\title{
The dynamics of the manifestations of atopic dermatitis in severe persistent uncontrolled asthma children with omalizumab therapy
}

\author{
Elena Vishneva*, Leyla Namazova-Baranova, Anna Alekseeva, Kamilla Efendieva, Anna Tomilova, \\ Natalia Voznesenskaya, Konstantin Volkov, Julia Levina
}

From EAACI Skin Allergy Meeting 2014

Krakow, Poland. 18-20 September 2014

\section{Background}

in children with severe persistent uncontrolled asthma atopic dermatitis (AD) is often characterized by persistent continually relapsing.

According to the approach ICON for children $>6$ y o with severe uncontrolled asthma as an additional treatment from 3-4 step of therapy omalizumab (Omab) is recommended for use of. We report on the experience of the use of Omab in children with asthma and AD.

\section{Methods}

Medical files of 64 children treated with Omab were analysed: $36 \%$ of patients have had a concomitant diagnosis of $A D, 39.1 \%$ of them suffered of severe $A D$ (SCORAD >60-70) at baseline.

All of the following data were recorded, at baseline and after 6, 12 and 24 mo of Omab therapy: values of SCORAD, duration of periods of remission and periods of exacerbation of $\mathrm{AD}$, quality of life (on a scale CDLQI and $A C Q$ ), requirement to use of topical steroids and maintenance asthma therapy, PFTs values, symptom scores and control level (GINA, ACT).

\section{Results}

The characteristics of children with uncontrolled severe asthma and persistent $\mathrm{AD}$ were: mean age of the beginning of Omab therapy - 11.8 y, 60\% girls, mean SCORAD index 72 points, quality of life (questionnaire CDLQI mean 15,2), the requirement of topical steroids was high (mean $6.2 \mathrm{~g} /$ day of therapy), the duration of periods of remission were extremely short and periods of exacerbation of $\mathrm{AD}$ were more than 280 days/y. Initial maintenance asthma therapy was ICS (mean daily dose: $750 \mu$ g fluticasone) with LABA, asthma symptom control questionnaires (mean ACT: 7.5, mean ACQ 23), mean FEV1 76\%, mean PFT 69.7\%.

After prolonged Omab therapy following indices significantly improved: mean SCORAD 28, 17 and 10 points (after 6, 12 and 24 mo accordingly), mean CDLQI 9, 4, and 2.1; the use of topical steroids $2.9,1.6$ and $0.7 \mathrm{~g} / \mathrm{d}$, periods of $\mathrm{AD}$ remission were extended, asthma control (mean ACT: 19, mean ACQ 12), mean FEV1 96\%.

The effectiveness of treatment was directly correlated with the duration of therapy.

\section{Conclusions}

Omab therapy not only improves asthma control, it allows to reduce of the manifestations and rate of exacerbations of severe $\mathrm{AD}$ and has shown steroid saving effect.

Published: 11 March 2015

doi:10.1186/2045-7022-5-S1-P3

Cite this article as: Vishneva et al:: The dynamics of the manifestations of atopic dermatitis in severe persistent uncontrolled asthma children with omalizumab therapy. Clinical and Translational Allergy 2015 5(Suppl 1):P3. 